\title{
Effects of Neurexan on Stress-Induced Changes of Spectral EEG Power: A Double-Blind, Randomized, Placebo-Controlled, Crossover Exploratory Trial in Human Volunteers
}

\author{
Wilfried Dimpfel \\ Justus-Liebig-University, Giessen c/o NeuroCode AG, Wetzlar, Germany \\ Email: wilfried.dimpfel@pharma.med.uni-giessen.de
}

How to cite this paper: Dimpfel, W. (2019) Effects of Neurexan on Stress-Induced Changes of Spectral EEG Power: A DoubleBlind, Randomized, Placebo-Controlled, Crossover Exploratory Trial in Human Volunteers. World Journal of Neuroscience, 9 , 100-112.

https://doi.org/10.4236/wjns.2019.93007

Received: June 28, 2019

Accepted: July 22, 2019

Published: July 25, 2019

Copyright $\odot 2019$ by author(s) and Scientific Research Publishing Inc. This work is licensed under the Creative Commons Attribution International License (CC BY 4.0).

http://creativecommons.org/licenses/by/4.0/

\begin{abstract}
Neurexan is a multicomponent natural medicinal product used for stress-related symptoms such as nervous restlessness and insomnia. The present study investigated the efficacy of Neurexan on stress-induced changes of brainwave frequencies using quantitative analysis of spectral EEG power. Thirty healthy male and female volunteers were tested in a randomized, placebo controlled cross-over trial under conditions of relaxation and experimental stress-induction. Recording of the EEG was performed over four hours after drug administration. Data was analyzed using the proprietary fast dynamic EEG technology Neurocode-Tracking. Quantitative EEG revealed a task dependent increase of beta 2 power as a surrogate parameter of stress induced anxiety. The elevated frontotemporal beta2 power was ameliorated by intake of Neurexan during the second and third hour after administration. This indicates that Neurexan is effectively helping subjects to better cope with task-related stress situations.
\end{abstract}

\section{Keywords}

Stress, Anxiety, qEEG, Brain Map, Brainwave Frequency, Homeopathy, Neurexan, Nx4

\section{Introduction}

Treating nervousness, restlessness, anxiety, and insomnia remains difficult, due to their complex and various origins. A recent study revealed that more than half of the working age population experience some stress that is sometimes also related to burnout, exhaustion and accompanied with depression and/or anxiety 
[1]. Stress symptoms are constantly increasing in the last years [2]. Anti-depressants, anxiolytics and beta-blockers are widely used to control some of the physical symptoms of anxiety-related disorders. These medications are often associated with side effects and are not recommended for long-term use. Natural medications such as Neurexan are regarded as safe alternatives. Neurexan has been previously investigated for various stress-related symptoms: A randomized, placebo-controlled trial revealed that Neurexan diminishes significantly stress-induced salivary cortisol and plasma adrenaline in an acute stress setting [3]. Neurexan was more effective in the treatment of insomnia than valerian as demonstrated in an observational study [4]. Another observational study comparing with valerian-based preparations demonstrated that Neurexan is the more effective treatment of nervousness and restlessness [5].

Neurophysiological recordings during different emotional states are well established. The electrical activity of the human brain was successfully used for characterization of drug effects for a long time [6] and more recently proved to be particularly useful for characterization of herbal preparations [7]. Task-related changes of spectral frequencies during mental loads are well known [8]. Quantitative Electroencephalogram (EEG) measurements were also suggested to replace cognitive testing in certain setups [9] and drug-induced changes of brain activity have been successfully evaluated by quantitative EEG measurements in the relaxed state and during the performance of cognitive tests [10]. In the present study, we investigated the effects of Neurexan on neurophysiological recordings during experimentally induced stress in healthy volunteers.

\section{Material and Methods}

\subsection{Trial Design and Medication}

This was a randomized, double-blind, placebo-controlled, crossover exploratory trial conducted from January to July 2006 in Germany, EudraCT No. 2005-003942-32. The study consisted of two experimental days with a washout period of at least one week in between, to exclude the presence of any "carry-over effects". The study was carried out in accordance with the Declaration of Helsinki (2000) on human rights and was approved by the local ethics committee and the German Federal Institute for Drugs and Medical Devices (Bundesinstitut für Arzneimittel und Medizin-produkte (BfArM), Bonn). All subjects gave their written consent prior to the study.

The subjects were randomly assigned to a treatment sequence, receiving either 4 tablets of the verum Neurexan on the first experimental day and 4 tablets of the placebo on the second experimental day, or vice versa. Neurexan is a medicinal product of Passiflora incarnata (purple passion flower) D2 $0.6 \mathrm{mg}$, Avena sativa (common oats) D2 $0.6 \mathrm{mg}$, Coffea arabica (coffee) D12 $0.6 \mathrm{mg}$, and Zincumisovalerianicum (valerianate of zinc) D4 $0.6 \mathrm{mg}$ in magnesium stearate 1.5 $\mathrm{mg}$ and lactose monohydrate $300 \mathrm{mg}$ marketed by Biologische Heilmittel Heel $\mathrm{GmbH}$, Baden-Baden, Germany. The respective placebo contained lactose mo- 
nohydrate, magnesium stearate and was identical in terms of size, color, taste, and labeling. Study medication and placebo were provided by Biologische Heilmittel Heel GmbH (Baden-Baden, Germany).

\subsection{Participants and Sample Size Calculations}

Due to the exploratory nature of this study, no power calculations were conducted. A convenience sample of 30 subjects ( $50 \%$ male and female) was defined as a recruitment target, which was achieved when 35 people volunteered. All male and female subjects were between 30 and 60 years old (average of $42.61 \pm$ 6.48 years, male $40.71 \pm 0.41$ and female $44.50 \pm 0.43$ ) and regarded themselves healthy. Participants reporting neurological disturbances of the central nervous system (CNS) using DSM-III criteria or a history of drug or ethanol abuse or participation in another study within the last six months were found not eligible and eventually excluded from the study. This was reviewed by a general medical examination before entering the trial.

\subsection{Experimental Procedure and Interventions}

Volunteers underwent a basic medical examination consisting of a physical examination, blood analysis, ECG and EEG before entering the trial. On the day of the examination, no beverages containing caffeine were allowed within the last 12 hours preceding the EEG recording. It was also ensured that they were not under the influence of alcohol or drugs during the experiments. The subjects were familiarized with the tests during the initial period of the session. Four did not show up for the experimental session and one had a pathological EEG. Therefore, data from 30 subjects (males and females, 1:1) were analyzed. All participants were informed about the goals of the study in detail and provided written informed consent.

Subjects were sitting alone in a quiet separate room in a comfortable easy chair. Baseline recording of $6 \mathrm{~min}$ under the condition of "eyes open" took place before ingestion of the trial medication. The next recording period took place during the performance of a mathematical task (validated test) with optical feedback on their performance by an online bar showing financial award or punishment. All recordings were repeated in hourly intervals up to 4 hours after the administration of the study medication. In the sessions, the subjects spent their time in the facility's break room. All individual experiments were initiated at the same time of day to exclude the potential influence of circadian rhythms on the EEG.

\subsection{Statistical Methods}

Due to the non-Gaussian distribution of EEG data, median values were evaluated, and the nonparametric sign test was chosen for comparison between Neurexan and placebo. No adjustments for multiple comparisons were made. Exploratory p-values are presented at the appropriate sites. Guidelines for the conduction of Pharmaco-EEG studies were followed [11]. 


\subsection{EEG Recording}

The EEG was recorded bipolarly from 17 surface electrodes according to the international 10/20 system with $\mathrm{Cz}$ as physical reference electrode (common average reference against all other electrodes) with the Computer-Aided-TopographicalElectro-Encephalo-Metry $\left(\right.$ CATEEM $\left.^{\circledR}\right)$ system from MEWICON CATEEM-Tec $\mathrm{GmbH}$ (Schwarzenberg, Austria) as described by Dimpfel et al., 2014 [12].

The Electrodes were positioned in the Central (C), Frontal (F), Parietal (P), Temporal $(\mathrm{T})$ and Occipital $(\mathrm{O})$ brain regions, even numbers represent the right hemisphere, uneven numbers the left hemisphere and $\mathrm{z}$ representing the "central" position (Figure 1).

In order to obtain information located in between the physically recorded locations, signals from 82 additional virtual electrodes were calculated to provide high-resolution topographical maps using a Lagrange interpolation. EEG was processed in a surface charge mode obtained by Laplacian estimates also known as current source density analysis [13]. The quantitative evaluation of the EEG signals was based on the measurement of absolute voltage changes, given in $\mu \mathrm{V}^{2}$.

The signals of all 99 electrode positions ( 17 real and 82 virtual) underwent the Fast Fourier Transformation (FFT) based on 364 ms lasting sweeps of data epochs (Hanning window). Data were analyzed from 1.375 to $35 \mathrm{Hertz}(\mathrm{Hz})$ using the CATEEM ${ }^{\circledR}$ software. For the final analysis using the fast dynamic mode, EEG frequency ranges were slightly adjusted to give the exact ranges for delta (red) 1.375 - 4.125, theta (orange) 4.125 - 6.875, alpha1 (yellow) 6.875 - 9.625, alpha2 (green) 9.625 - 12.375, beta1 (turquoise) 12.375 - 17.875 and beta2 (blue) 17.875 - 34.375 .

This adjustment was a precondition for the fast dynamic frequency analysis (Neurocode-Tracking [12]) because under this condition each frequency range from delta up to alpha2 only contains one frequency, the middle frequency within the particular frequency band. This frequency analysis was based on absolute spectral power values.

An automatic artefact rejection of the CATEEM ${ }^{\circ}$-System was used that eradicates EEG alterations caused by eye-blinks, swallowing, respiration, etc. during

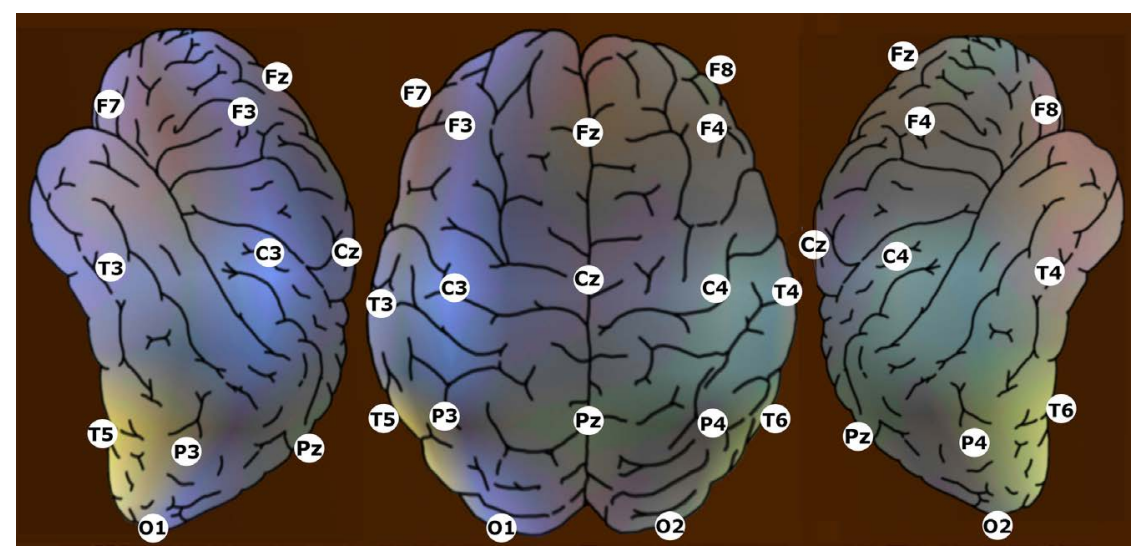

Figure 1. Electrode Positions. 
the recording. Artefact rejection was individually adjusted by the investigator. For each subject, the adjustment was kept constant throughout the experimental sessions.

Color-coding of the maps was achieved by transforming spectral power into spectral colors followed by additive color mixture according to the so-called Red-Green-Blue-mode (RGB). Thus, maps represent a true result of the spectral changes and are not constructed as a so-called "false color coding". For documentation of the drug effects, differential maps were constructed using Placebo data as $100 \%$ reference. The graphs show the relative, time-averaged changes in electric brain activity of each specific recording condition (\% change with respect to the reference placebo). Absolute power values from the baseline recording were set to $100 \%$ and all data were expressed in $\%$ of these baseline values.

\subsection{Profile of Mood States}

To be able to assess subjective feelings and to better understand and interpret the results subjects rated themselves according to the Profile of Mood States (POMS) assessing six dimensions of the mood construct, anger, confusion, depression, fatigue, tension, and vigor in a shortened German version [14]. Sub-scores were calculated for displeasure, energy, tiredness and depression. Ratings were captured before baseline recording and after finishing the electrophysiological session.

\section{Results}

\subsection{Effects on Spectral Power under the Recording Condition "Eyes Open"}

Under the condition of Eyes Open (EO; relaxed before the stress induction), spectral power in the EEG was very similar at baseline before intake of drugs for both treatment arms. For the documentation of drug-induced changes of the spectral pattern, Placebo results were set to $100 \%$ for all time points and electrode positions and the results for Neurexan were given as percentage change. Results are shown in Figure 2. The colored bars indicate the spectral power within 6 frequency ranges, red $=$ delta, orange $=$ theta, yellow $=$ alphal, green $=$ alpha2, turquoise $=$ beta 1 and blue $=$ beta2, and the solid line is indicating the Placebo value. The differences already at baseline at electrode positions $\mathrm{F}_{3}, \mathrm{~F}_{8}$ and $\mathrm{T}_{4}, \mathrm{~T}_{6}$ are regarded of minor importance in respect to effect-size for the parameter. Relevant effects of Neurexan emerge during the second hour after intake. During this period significant attenuation of spectral power is seen predominantly in $\mathrm{P}$ and $\mathrm{T}$ areas. Mainly alpha and beta power were affected. A similar pattern of attenuation of spectral power within the same brain regions developed during the third hour. During the last hour, statistically significant attenuations of spectral power were still detected in $\mathrm{F}, \mathrm{T}$ and $\mathrm{P}$ brain areas.

Brain Maps as depicted in Figure 3 represent a graphical interpretation of drug-induced changes of the spectral pattern. Subtle drug effects can be seen 
$\Delta \Phi C_{2} \Delta \Phi F_{2} \Delta \Phi F_{3} \Delta \Phi C_{3} \Delta \Phi P_{3} \Delta \Phi P_{2} \Delta \Phi P_{4} \Delta \Phi C_{4} \Delta \Phi F_{4} \quad \Delta \Phi F_{7} \Delta \Phi T_{3} \Delta \Phi T_{5} \Delta \Phi O_{1} \quad \Delta \Phi 0_{2} \quad \Delta \Phi T_{6} \Delta \Phi T_{4} \Delta \Phi F_{8} \quad 120 \%$ N

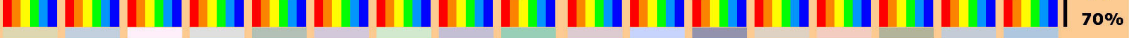

\section{$1 \mathrm{~h}$}

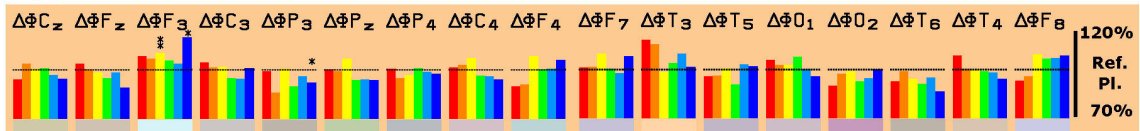

$2 h$

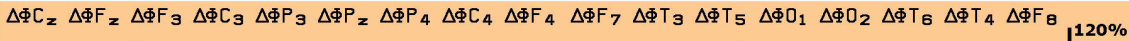

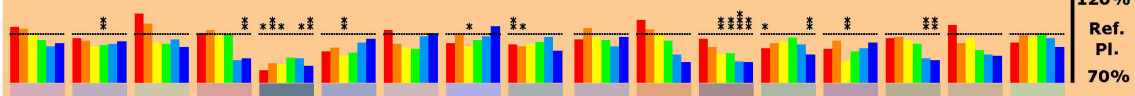

$3 h$

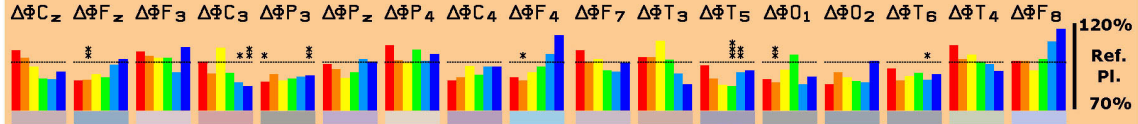

$4 \mathrm{~h}$

$\Delta \Phi C_{2} \Delta \Phi F_{2} \Delta \Phi F_{3} \Delta \Phi C_{3} \Delta \Phi P_{3} \Delta \Phi P_{2} \Delta \Phi P_{4} \Delta \Phi C_{4} \Delta \Phi F_{4} \Delta \Phi F_{7} \Delta \Phi T_{3} \Delta \Phi T_{5} \Delta \Phi O_{1} \Delta \Phi O_{2} \Delta \Phi T_{6} \Delta \Phi T_{4} \Delta \Phi F_{8}{ }_{120 \%}$

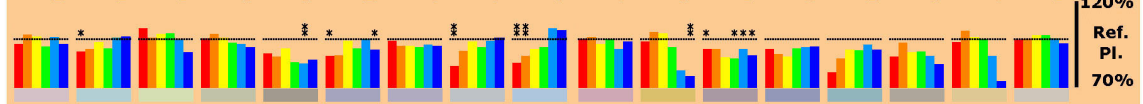

Figure 2. Time dependence of regional development of spectral power within 6 frequency ranges for the recording condition "EO". $h=$ hours. Statistically significant differences of Neurexan (Verum) in comparison to Placebo $(\mathrm{Pl})$ are marked by asterisks: ${ }^{\star} \mathrm{p}<0.05,{ }^{\star \star} \mathrm{p}$ $<0.01$ and ${ }^{* * *} \mathrm{p}<0.001$.

already during the first hour after intake. Greater differences between Neurexan and Placebo are seen during the second and third hour. There are still frequency changes during the last hour when compared to the baseline at 0 hour before the intake of Neurexan. The maps uncover a clearly time-dependent action of Neurexan.

To see which frequency ranges would mainly indicate the action of Neurexan, data from all electrode positions were averaged to give a median value. Values recorded before the intake of the drugs were set to $100 \%$. Drug-induced changes are depicted in \% of these baseline values for all hours after the intake as given in Figure 6. There were no significant drug effects with respect to slow frequencies delta and theta. However, there is a clear attenuation of alpha waves induced by Neurexan, which partially becomes statistically significant in comparison to Placebo during the second (alpha1) and third hour (alpha2). With respect to beta spectral power, generally lower values were detected in the presence of Neurexan.

\subsection{Effects on Spectral Power under the Recording Condition "Concentration Performance Test"}

The second recording condition consisted in the measurement of electric activity during the performance of a Concentration Performance Test (CPT). Increased values in comparison to relaxation (condition EO) were observed on theta and 


\section{Placebo v.s. Verum}
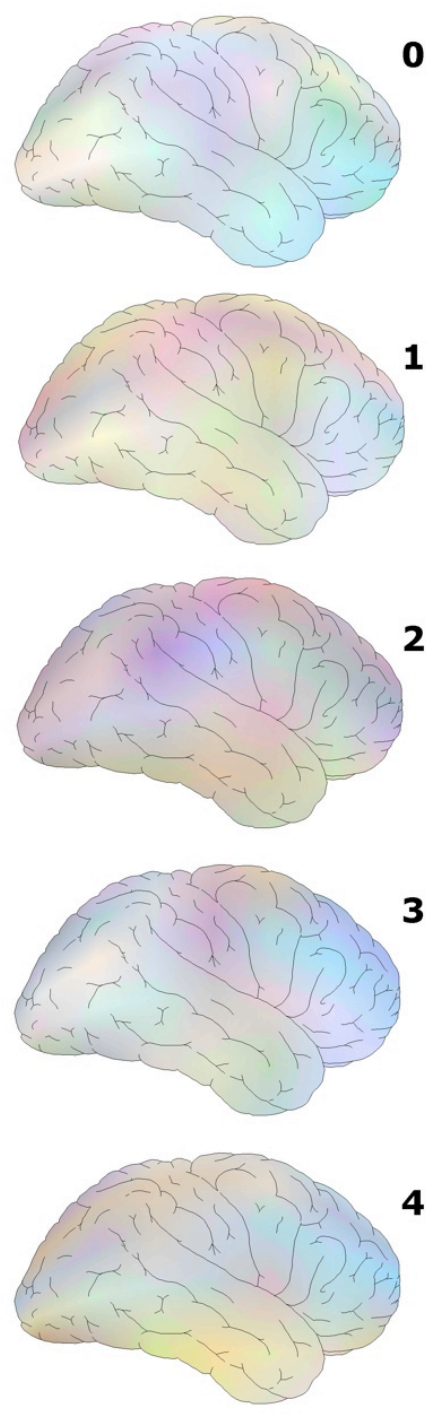

3h

\section{Eyes Open}

Oh

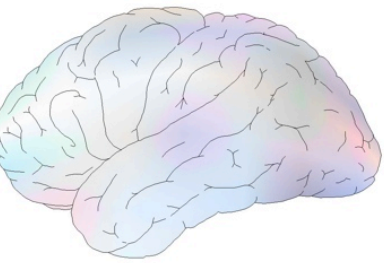

1h

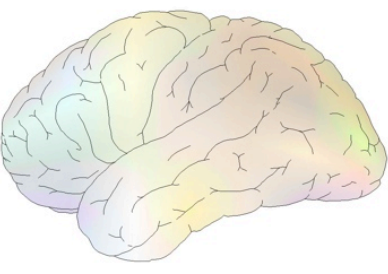

$\mathbf{2 h}$
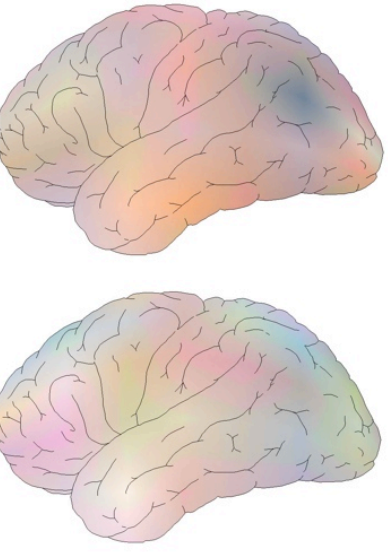

4h

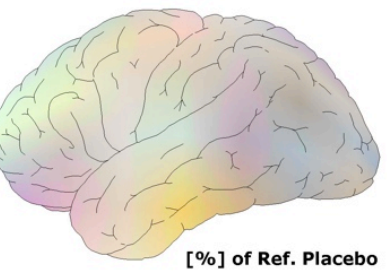

Figure 3. Time-dependent comparison between Placebo and Neurexan (Verum), illustrated as spectral maps for recording condition "EO". Spectral patterns are given in \% of Placebo. Front end of the brain is oriented towards the middle of the image. $h=$ hours after intake. Left part of the image represents right hemishere, right part of the image represents left hemisphere.

beta2 power at electrode positions $\mathrm{F}_{7}$ and $\mathrm{F}_{8}$ that was due to the performance of mental work. Placebo and Neurexan groups were equally affected.

As also seen at the EO condition, some minor differences between Neurexan and Placebo in spectral power at CPT were already detected during the baseline recording at 0 hours before intake of the drugs (Figure 4 ). The differences between Neurexan and Placebo remained of minor importance one hour after drug administration in respect to effect-size for the respective parameter. The effect of Neurexan became relevant 2 hours after intake where great and statistically significant effects emerged. The dominant effect consisted with a general attenuation of frontotemporal beta power, that reached statistical significance compared to 
$1 \mathbf{h}$

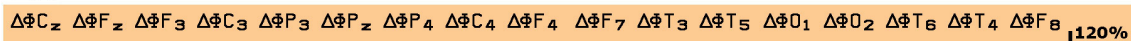
(1)

2h

$\Delta \Phi \mathrm{C}_{\mathbf{z}} \Delta \Phi \mathrm{F}_{\mathbf{z}} \Delta \Phi \mathrm{F}_{3} \Delta \Phi \mathrm{C}_{3} \Delta \Phi \mathrm{P}_{3} \Delta \Phi \mathrm{P}_{\mathbf{z}} \Delta \Phi \mathrm{P}_{4} \Delta \Phi \mathrm{C}_{4} \Delta \Phi \mathrm{F}_{4} \Delta \Phi \mathrm{F}_{7} \Delta \Phi \mathrm{T}_{3} \Delta \Phi \mathrm{T}_{5} \Delta \Phi 0_{1} \Delta \Phi 0_{2} \Delta \Phi \mathrm{T}_{6} \Delta \Phi \mathrm{T}_{4} \Delta \Phi \mathrm{F}_{8}{ }_{120 \%}$

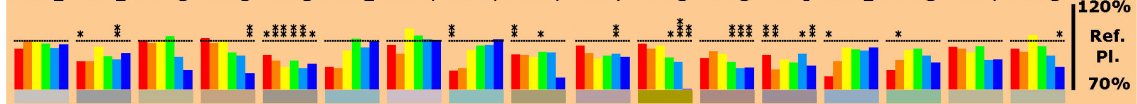

\section{3h}

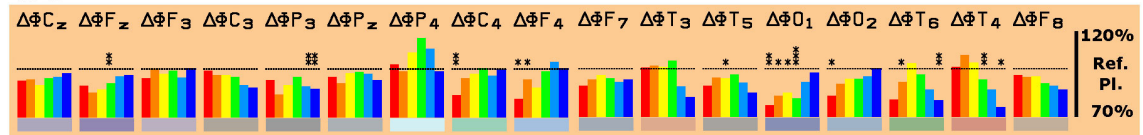

4h

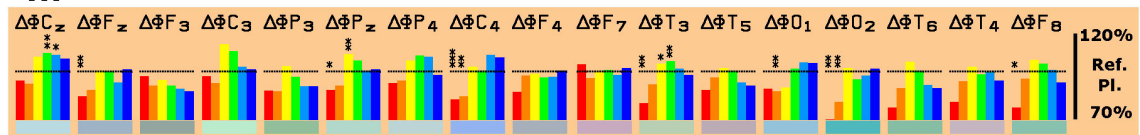

Figure 4. Time dependence of regional development of spectral power within 6 frequency ranges for the recording condition "CPT". $h=$ hours. Statistically significant differences of Neurexan in comparison to Placebo $(\mathrm{Pl})$ are marked by asterisks: ${ }^{*} \mathrm{p}<0.05,{ }^{* *} \mathrm{p}<0.01$ and ${ }^{* *} \mathrm{p}<0.001$.

Placebo at several brain regions (electrode positions $F_{z}, F_{7}, F_{8}, T_{3}, T_{5}$ ). Three hours after drug administration, the pattern was comparable to the 2 hours pattern. Temporal beta power was still significantly attenuated but was more limited to positions $\mathrm{T}_{4}(\mathrm{p}<0.05)$ and $\mathrm{T}_{6}(\mathrm{p}<0.01)$.

In the visualization of spectral power in the brain regions, given in the brain maps in Figure 5, Neurexan and Placebo are barely distinguishable at baseline ( 0 h). Already at the 1 hour time point distinct patterns can be recognized and 2 hours after drug administration, the Neurexan map differs very clearly. Distinguishable maps from the baseline were also observed at the 3 hours timepoint, but differences ceased during the last hour.

Averaging of the data from all electrode positions in the presence of mental work and setting the baseline values to $100 \%$ reveals frequency and time dependent drug effects. Comparing spectral power in the presence of Neurexan or Placebo during several hours after intake uncovered, that major statistically significant effects occurred with respect to beta1 and beta2 spectral power (Figure 6). Neurexan led to a significant attenuation of the elevated beta power during the first 3 hours after drug administration.

\subsection{Behavioral Scores and Medical Examination}

Analysis of the POMS data showed some minor changes during the course of the 
Placebo v.s. Verum
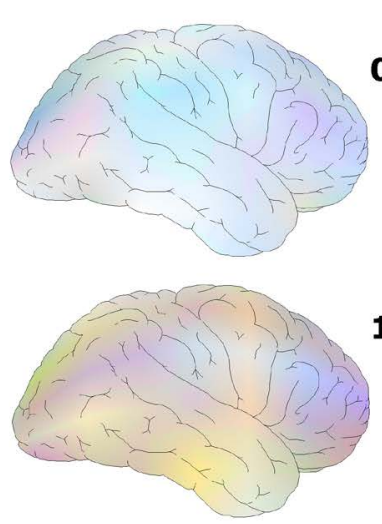

$1 \mathbf{h}$
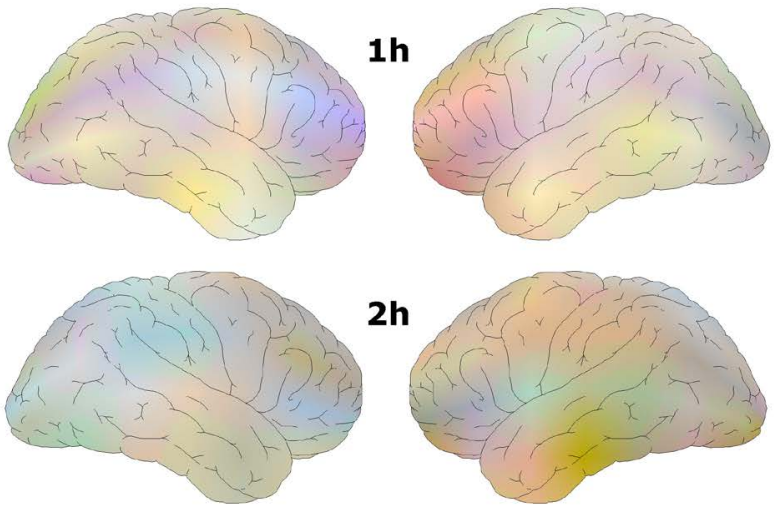

$\mathbf{2 h}$
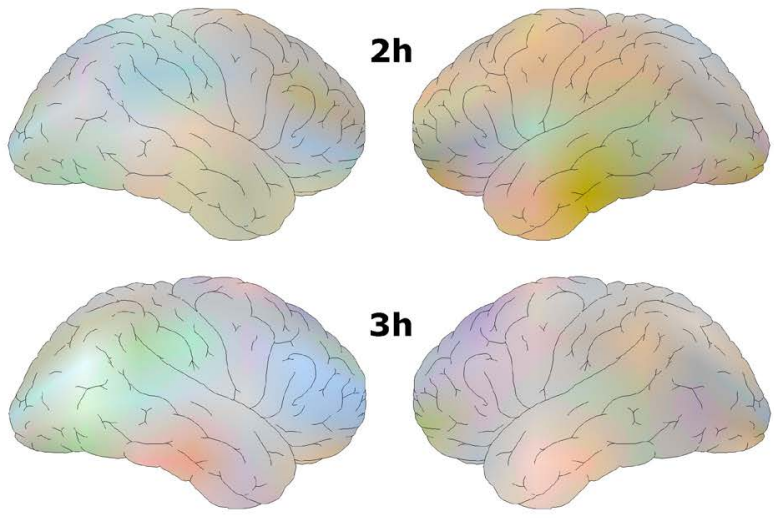

3h
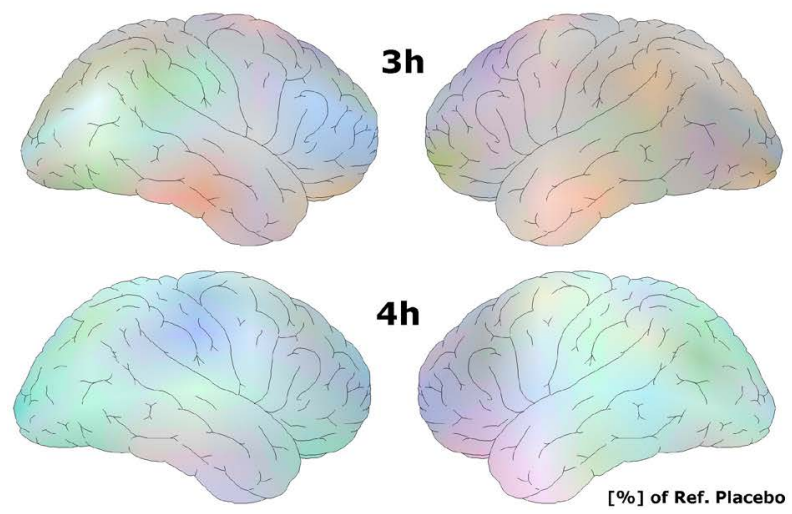

Figure 5. Time-dependent comparison between Placebo and Neurexan, illustrated as spectral maps for recording condition "CPT". Spectral patterns are given in \% of Placebo. Front end of the brain is oriented towards the middle of the image. $\mathrm{h}=$ hours. Left part of the image represents right hemishere, right part of the image represents left hemisphere.

trial, however, equally in Neurexan and Placebo groups. No statistically significant effect of Neurexan compared to Placebo on POMS was observed. Also, the analysis of the quality of mathematical calculations applied here via calculations of the percentage of the correct results, did not change in the presence of Neurexan in comparison to Placebo.

In regard to the medical examination, no relevant differences between Placebo and Neurexan were detected. Subjects generally reported very good tolerability. Tiredness was reported in seven instances during Neurexan treatment and in three instances during Placebo treatment. Headaches were reported in two cases during Neurexan and one case during Placebo treatment. No changes in blood parameters (complete blood count plus glutamate-oxaloacetate-transaminase, glutamate-pyruvate-transaminase, gamma-glutamyl-transferase, creatinine, glucose, and sodium) were observed. 


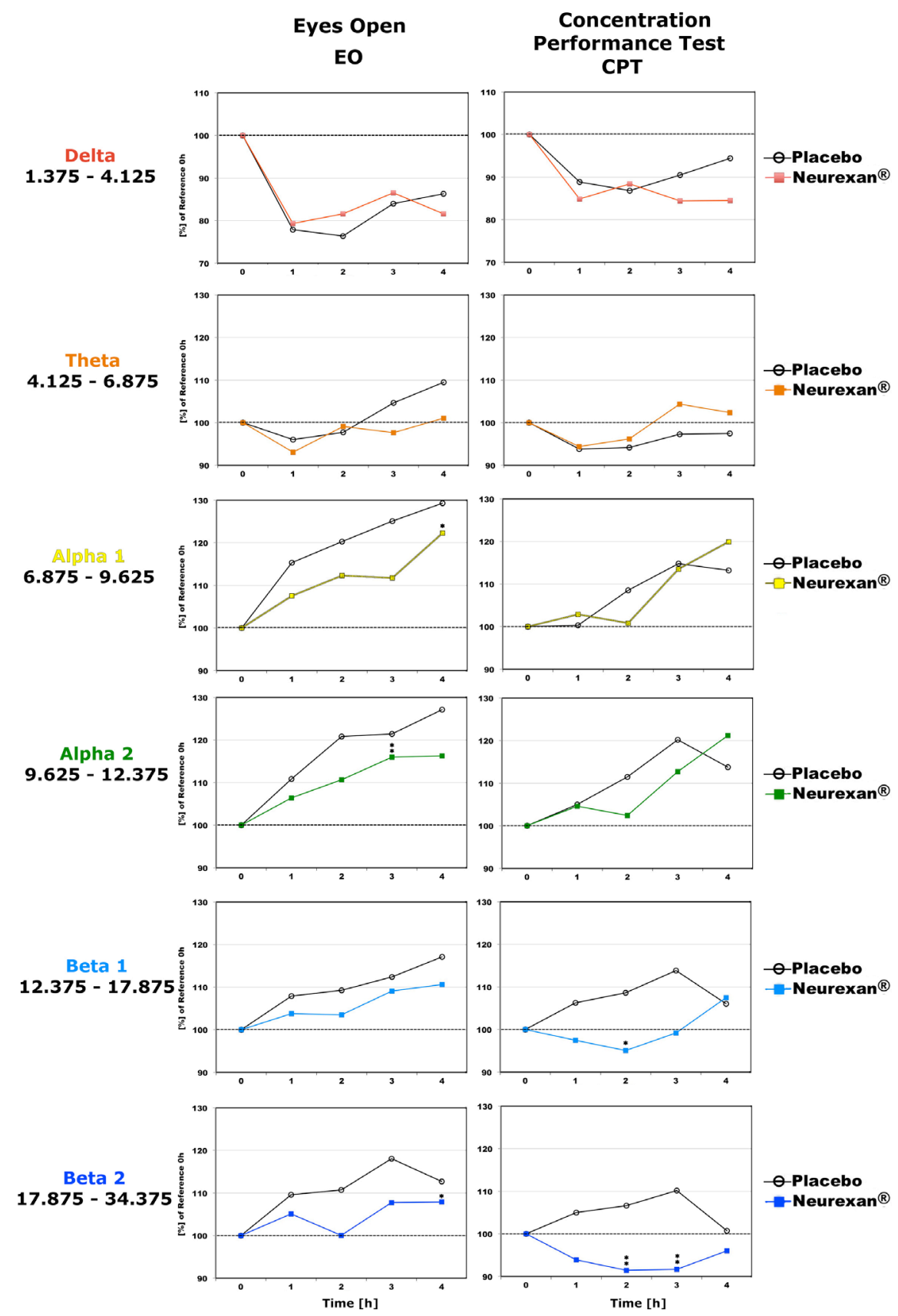

Figure 6. Time-dependent changes of spectral frequency content for the recording conditions "EO" (left panel) and "CPT" (right panel). The colored lines represent the time-dependent, hour 0 to hour 4 , changes after four tablets of Neurexan were administered. The grey lines represent the Placebo group. Statistically significant differences are marked with ${ }^{\star} \mathrm{p}<0.05$ and ${ }^{\star *} \mathrm{p}<0.01$.

\section{Discussion}

Natural medications such as Neurexan have been proposed for the treatment of stress-related disorders as safe alternatives to conventional medications due to anticipated better tolerability with fewer side effects for long term use. Neurexan has been successfully used for various stress and related symptoms [3], insomnia 
[4] nervousness and restlessness [5] measuring various endpoint variables. In the present study, it was demonstrated, that Neurexan effectively modulates brainwave frequencies using quantitative analysis of spectral EEG power.

The principal finding of the study is the observation that stress-induced increase of frontotemporal spectral beta2 power during the performance of a cognitive task was largely prevented by Neurexan from the second hour of administration onwards. Additionally, a blunted circadian increase of spectral alpha2 power was observed.

The target variable related to the induced cognitive stress represents a surrogate parameter. It has been shown, however, that an increase of spectral beta power from the EEG is connected to a strong emotional environment in humans [15] [16]. Preclinical and clinical neurophysiological studies investigated changes in brain activity, in which spectral beta2 power seems to be related to GABAergic transmission [6].

In regard to the blunted circadian increase of spectral alpha2 power, preclinical experiments have shown that alpha 2 frequencies change with a modulation of dopaminergic transmission, and this frequency is related to working memory in humans [7] [17], and [18]. The frequency decreases during the performance of cognitive tasks, in which high mental concentration is needed, and mainly temporal and parietal brain regions are involved [8].

Taken together the results suggest a dual effect of Neurexan: Stress reduction as given by the attenuation of beta2 spectral power during stress-induction and the prevention of circadian loss in the ability to concentrate as given by the decreased circadian increase of spectral alpha2 power. However, one must be aware of the fact that we are regarding only so-called surrogate parameters of stress.

Certainly, further studies are required to evaluate a long-term efficacy of Neurexan such as a potential general increase of stress resilience towards a better ability to concentrate in comparison to placebo (changes during circadian rhythm) [19]. However, the results are promising that Neurexan could be prescribed as a non-sedative first line treatment for stress-related conditions. Neurexan may help patients suffering from stress-related symptoms to better cope with stress situations and to better keep the focus during the day. That might in further consequence also prevent from often accompanied symptoms like burnout, exhaustion, insomnia, depression, and anxiety.

\section{Acknowledgements}

The Study was financially supported by Biologische Heilmittel Heel GmbH, D-76532 Baden-Baden, Germany. The help of Dr. Stephan Duller in preparing the manuscript is highly appreciated.

\section{Conflicts of Interest}

There was no conflict of interest. 


\section{References}

[1] Wiegner, L., et al. (2015) Prevalence of Perceived Stress and Associations to Symptoms of Exhaustion, Depression and Anxiety in a Working Age Population Seeking Primary Care-An Observational Study. BMC Family Practice, 16, 38. https://doi.org/10.1186/s12875-015-0252-7

[2] European Risk Observatory Report (2009) OSH in Figures: Stress at Work Facts and Figures. https://osha.europa.eu/en/tools-and-publications/publications

[3] Doering, B.K., et al. (2016) Effects of Neurexan in an Experimental Acute Stress Setting-An Explorative Double-Blind Study in Healthy Volunteers. Life Sciences, 146, 139-147. https://doi.org/10.1016/j.lfs.2015.12.058

[4] Waldschutz, R. and Klein, P. (2008) The Homeopathic Preparation Neurexan vs. Valerian for the Treatment of Insomnia: An Observational Study. The Scientific World Journal, 8, 411-420. https://doi.org/10.1100/tsw.2008.61

[5] Hubner, R., van Haselen, R. and Klein, P. (2009) Effectiveness of the Homeopathic Preparation Neurexan Compared with That of Commonly Used Valerian-Based Preparations for the Treatment of Nervousness/Restlessness-An Observational Study. The ScientificWorld Journal, 9, 733-745. https://doi.org/10.1100/tsw.2009.95

[6] Saletu, B., Grunberger, J. and Linzmayer, L. (1983) Quantitative Pharmaco-EEG and Performance after Administration of Brotizolam to Healthy Volunteers. British Journal of Clinical Pharmacology, 16, 333S-345S.

https://doi.org/10.1111/j.1365-2125.1983.tb02308.x

[7] Dimpfel, W., Todorova, A. and Vonderheid-Guth, B. (1999) Pharmacodynamic Properties of St. John's Wort-A Single Blind Neurophysiological Study in Healthy Subjects Comparing Two Commercial Preparations. European Journal of Medical Reserach, 4, 303-312.

[8] Schober, F., Schellenberg, R. and Dimpfel, W. (1995) Reflection of Mental Exercise in the Dynamic Quantitative Topographical EEG. Neuropsychobiology, 31, 98-112. https://doi.org/10.1159/000119179

[9] Corradini, P.L. and Persinger, M.A. (2015) Replace Psychometric Inferences with Direct Brain Measurements: LORETA Reflects Traditional Cerebral Loci for Neuropsychological Tests. Neuroscience and Medicine, 6, 107-115. https://doi.org/10.4236/nm.2015.630187

[10] Dimpfel, W., Koch, K. and Weiss, G. (2012) Single Dose Effects of Pascoflair on Current Source Density (CSD) of Human EEG. Neuroscience \& Medicine, 3, 130-140. https://doi.org/10.4236/nm.2012.32018

[11] Ferber, G., et al. (1999) IPEG Guideline on Statistical Design and Analysis for Pharmaco-Dynamic Trials. Neuropsychobiology, 39, 92-100.

https://doi.org/10.1159/000026567

[12] Dimpfel, W. and Hofmann, H.C. (2014) Neurocode-Tracking Based on Quantitative Fast Dynamic EEG Recording in Combination with Eye-Tracking. World Journal of Neuroscience, 4, 106-119. https://doi.org/10.4236/wjns.2014.42013

[13] Dimpfel, W., et al. (1996) Source Density Analysis of Functional Topographical EEG: Monitoring of Cognitive Drug Action. European Journal of Medical Research, 1, 283-290.

[14] Grulke, N., et al. (2006) Standardization of the German Short Version of "Profile of Mood States" (POMS) in a Representative Sample-Short Communication. Psychotherapie, Psychosomatik, Medizinische Psychologie, 56, 403-405.

[15] Schutter, D.J., et al. (2001) Parietal Electroencephalogram Beta Asymmetry and Se- 
lective Attention to Angry Facial Expressions in Healthy Human Subjects. Neuroscience Letters, 314, 13-16. https://doi.org/10.1016/S0304-3940(01)02246-7

[16] Dimpfel, W., Wedekind, W. and Keplinger, I. (2003) Gender Difference in Electrical Brain Activity during Presentation of Various Film Excerpts with Different Emotional Content. European Journal of Medical Research, 8, 192-198.

[17] Dimpfel, W. (2008) Pharmacological Modulation of Dopaminergic Brain Activity and Its Reflection in Spectral Frequencies of the Rat Electropharmacogram. Neuropsychobiology, 58, 178-186. https://doi.org/10.1159/000191124

[18] Stipacek, A., et al. (2003) Sensitivity of Human EEG Alpha Band Desynchronization to Different Working Memory Components and Increasing Levels of Memory Load. Neuroscience Letters, 353, 193-196.

https://doi.org/10.1016/j.neulet.2003.09.044

[19] Cummings, L., Dane, A., Rhodes, J., Lynch, P. and Hughes, A.M. (2000) Diurnal Variation in the Quantitative EEG in Healthy Adult Volunteers. British Journal of Clinical Pharmacology, 50, 21-26. https://doi.org/10.1046/j.1365-2125.2000.00205.x 